\title{
INJECTIVE REPRESENTATIONS OF INFINITE QUIVERS. APPLICATIONS
}

\author{
E. ENOCHS, S. ESTRADA AND J.R.GARCÍA ROZAS
}

\begin{abstract}
In this article we study injective representations of infinite quivers. We classify the indecomposable injective representations of trees and then describe Gorenstein injective and projective representations of barren trees.
\end{abstract}

\section{INTRODUCTION}

The major impetus for the study of the representations of quivers was given by Gabriel's study of the finite quivers of finite representation type (see [1]) and their connection with the Dynkin diagrams associated with finite dimensional semisimple Lie algebras over the field of complex numbers.

The classical representation theory of quivers involved finite quivers and assumed that the ring was an algebraically closed field with the assumption that all vector spaces involved were finite dimensional. In [14 the study of semisimple representations of these kinds of quivers were considered. Recently representations by modules over more general quivers have been studied. Our concern will be these kinds of representations and is a continuation of the program initiated in [7] and continued in [6], [5], [2] and [9].

Many categories of graded modules over graded rings are equivalent to representations of quivers which are often infinite. For a simple example, the category of $\mathbb{Z}$-graded modules over

Key words and phrases.

The authors are partially supported by the DGI MTM2005-03227.

Estrada's work was supported by a MEC/Fulbright grant from the Spanish Secretaría de Estado de Universidades e Investigación del Ministerio de Educación y Ciencia. 
the graded ring $R[x]=R+R x+R x^{2}+\cdots$ (here $R$ is any ring with identity) is equivalent to the category of representations over $R$ of the infinite line quiver. Less trivial examples can be given involving group rings $R[G]$ with the obvious grading. In [3] it was shown that the category of quasi-coherent sheaves over any scheme is equivalent to a category of representations of a quiver (with certain modifications on the representations). In many of these cases the quiver viewpoint leads to simplifications of proofs and of the descriptions of objects in related categories. This has certainly proved true in the case of finite quivers and promises to be so when the quivers are infinite.

Our techniques are necessarily different from the usual ones concerning quivers without oriented cycles, and in general for the classic treatment of representation theory of associative algebras (see for example [1]). We consider the geometric properties of the quiver and also have used infinite matrix techniques to classify projective representations (see [2]). In [9] flat representations were studied and in [6] the so called noetherian quivers were characterized.

So our main concern in this paper is the study of injective representations over a possibly infinite quiver in terms of local properties of the representations (see properties $i$ ) and $i i$ ) of Proposition 2.1). As we will see in Section 6 these local properties turn to be very useful in studying and characterizing Gorenstein injective, projective and flat representations of quivers. These are of particular interest for defining a version of relative homological algebra that is called Gorenstein homological algebra. For it has been recently proved in [5] (by using the results of [4]) that a fruitful version of Gorenstein homological algebra can be developed in the category of representations over an arbitrary quiver, when the base ring is Gorenstein. Moreover by [4] we get that two model structures can be derived in the category of representations over a quiver 
where we use these Gorenstein injective and Gorenstein projective representations to define the cofibrations (see [12] for a deep study on model category structures).

On the other hand, infinite and barren trees (see Section 5) appear naturally in the study of finiteness conditions in the category of representations of quivers. In particular concerning the question of when this category admits a family of noetherian generators (cf. [6]) and in the characterization of a Gorenstein path ring (cf. [5]). So in Section 3 we study indecomposable injective representations of trees to determinate the structure of injective representations on barren trees (see the remark after Corollary [5.5).

Sections 4 and 5 are devoted to exhibiting a wide class of quivers whose injective representations admit the previous "local" characterization (the so called source injective representation quivers, see Definiton 2.2.). One interesting question is to ask if this characterization carries over to quivers with relations. For instance, in [10] it is proved that this is the case when we consider the infinite line quiver on both sides and then take the monomial relation given by the composition of $N$-paths. So we get a local structure theorem for injective representations in the category of $N$-complexes of modules $(N \geq 2))$ and all machinery of Section 6 can be applied in this situation.

\section{PRELiminaries}

Throughout this article we will use the terminology and results of [7].

We keep the notation introduced in [1]. All rings considered in this paper will be associative with identity and, unless otherwise specified, not necessarily commutative. The letter $R$ will usually denote a ring. 
As usual we denote a quiver by $Q$ with the understanding that a quiver is a fourtuple $Q=$ $(V(Q), \Gamma(Q), s, t)$ where $V(Q)$ is a set of vertices, a set $\Gamma(Q)$ of arrows between these vertices and two maps $s, t: \Gamma(Q) \rightarrow V(Q)$, where for each $a \in \Gamma(Q), s(a)$ and $t(a)$ assign to an arrow $a$ the source vertex and terminal vertex of $a$ respectively. All quivers considered in this paper may be infinite, that is, one of the two sets $V(Q)$ or $\Gamma(Q)$ can be infinite. Note that we do not exclude loops or multiple arrows in the definition of a quiver. Sometimes we will denote $V(Q)$ (resp. $\Gamma(Q))$ simply as $V$ (resp. $\Gamma$ ) if the quiver is understood. A finite path $p$ of a quiver $A$ is a sequence of arrows $a_{n} \cdots a_{2} a_{1}$ with $t\left(a_{i}\right)=s\left(a_{i+1}\right)$ for all $i=0, \ldots, n-1$. Therefore $s(p)=s\left(a_{1}\right)$ and $t(p)=t\left(a_{n}\right)$. Two paths $p$ and $q$ of a quiver can be composed, getting another path $q p$ whenever $t(p)=s(q)$. A quiver can be thought as a small category where the objects are the vertices and the morphisms are the paths. The vertices of $Q$ can be considered as the identities of $Q$, that is, a vertex $v$ of $Q$ is a trivial path where $s(v)=t(v)=v$. A tree is a quiver $T$ having a vertex $v$ such that for another vertex $w$ of $T$ there exists a unique path $p$ such that $s(p)=v$ and $t(p)=v$. Such vertex $v$ is called the root of the tree $T$. A forest is a quiver in which every connected component is a tree. For a path $p$ of $Q$ we denote by $t(p)$ (resp. $s(p))$ the final (resp. the initial) vertex of $p$.

A representation by modules $\mathcal{X}$ of a given quiver $Q$ is a functor $\mathcal{X}: Q \rightarrow R$-Mod. Such a representation is determined by giving a module $\mathcal{X}(v)$ to each vertex $v$ of $Q$ and a homomorphism $\mathcal{X}(a): \mathcal{X}\left(v_{1}\right) \rightarrow \mathcal{X}\left(v_{2}\right)$ to each arrow $a: v_{1} \rightarrow v_{2}$ of $Q$. A morphism $\eta$ between two representations $\mathcal{X}$ and $\mathcal{Y}$ is a natural transformation, so it will be a family $\left\{\eta_{v}\right\}_{v \in V}$ such that $\mathcal{Y}(a) \circ \eta_{v_{1}}=\eta_{v_{2}} \circ \mathcal{X}(a)$ for any arrow $a: v_{1} \rightarrow v_{2}$ of $Q$. Thus the representations of a quiver $Q$ by 
modules over a ring $R$ is a category, denoted by $(Q, R$-Mod), which is a Grothendieck category with enough projectives.

The category $(Q, R$-Mod) is equivalent to the category of modules over the path algebra $R Q$, which is a ring with enough idempotents that in general does not have a unit (unless $|V|$ is finite).

For a given quiver one can find a family injective cogenerators from an adjoint situation as it is shown in [7]. For every vertex $v \in V$ and the embedding morphism $\{v\} \subseteq Q$ the family $\left\{e_{*}^{v}(E): v \in V\right\}$ is a family of injective cogenerators of $(Q, R$-Mod), whenever $E$ is an injective cogenerator of $R$-Mod. The functor $e_{*}^{v}: R$-Mod $\rightarrow(Q, R$-Mod) is defined in [7, Section 4] as $e_{*}^{v}(M)(w)=\prod_{Q(w, v)} M$, where $Q(w, v)$ denotes the set of paths $p$ in $Q$ such that $s(p)=w$ and $t(p)=v$. If $a: w_{1} \rightarrow w_{2}$ is an arrow, then

$$
e_{*}^{v}(M)(a): \prod_{Q\left(w_{1}, v\right)} M \rightarrow \prod_{Q\left(w_{2}, v\right)} M
$$

is given by the coordinate-wise function $e_{*}^{v}(M)(a)=\prod_{Q\left(w_{2}, v\right) a} i d_{Q\left(w_{2}, v\right)}$. Then by [7, Theorem 4.1] $e_{*}^{v}$ is the right adjoint functor of the evaluation functor $T_{v}:(Q, R$-Mod $) \rightarrow R$-Mod given by $T_{v}(\mathcal{X})=\mathcal{X}(v)$ for any representation $\mathcal{X} \in(Q, R$-Mod $)$.

We will need the property satisfied by injective representations given by the next result.

Proposition 2.1. Let $Q$ be any quiver and let $(Q, R$-Mod) the category of representations of $Q$ by left $R$-modules. If $\mathcal{X} \in(Q, R$-Mod $)$ is injective then:

i) $\mathcal{X}(v)$ is an injective $R$-module, for any vertex $v$ of $Q$.

ii) For any vertex $v$ the morphism

$$
\mathcal{X}(v) \rightarrow \prod_{s(a)=v} \mathcal{X}(t(a))
$$


induced by $\mathcal{X}(v) \rightarrow \mathcal{X}(t(a))$ is a splitting epimorphism.

Proof. We consider the injective representations $e_{*}^{v}(E)$ associated with a given vertex of $Q$ and an injective left $R$-module $E$. By the construction given in the proof of [7, Theorem 4.1] we see that each $e_{*}(E)$ has the property of the Proposition. As noted in [7, pg.303] the $e_{*}^{v}(E)$ (varying $v$ and $E$ ) cogenerate $(Q, R$-Mod). So any injective representation is a retract of products of the various $e_{*}^{v}(E)$ 's. The property is preserved under taking products and retracts and so we get the desired result.

Since the category of representations of a quiver $Q$ is always a Grothendieck category, it has enough injective representations. In this paper we are mainly concerned with studying when injective representations are characterized in terms of conditions $i$ ) and $i i$ ) of Proposition 2.1. These conditions involve local properties of the representation on the vertices and their corresponding sources, so they motivate the following definition which is pivotal for the rest of the paper.

Definition 2.2. We say that a quiver $Q$ is a source injective representation quiver if for any $R$ the injective representations of $(Q, R$-Mod) can be characterized in terms of conditions $i)$ and $i i)$ of Proposition 2.1. We will denote by $\mathfrak{I}$ the class of all source injective representation quivers.

One of the main goal of the paper is to study the class $\mathfrak{I}$ and more precisely to determine which trees belong to it. For this purpose, it is convenient to start Section 3 by determining the indecomposable injective representations of trees. As we will see in Section 6, there are important properties which can be derived from the fact that a quiver is in $\mathfrak{I}$. 
Thus in Section 4 we prove that a wide class of quivers, the so called right rooted quivers (that is those which do not contain a path of the form $\bullet \rightarrow \bullet \rightarrow \cdots$ ) are in $\mathfrak{I}$. But there are also important non-right rooted quivers in $\mathfrak{I}$, concretely, in Section 5 we prove that $A_{\infty}^{\infty}, A_{\infty}^{+}$and, more generally any barren tree are source injective representation quivers.

Of course there is a dual of Definition 2.2 by dualizing properties $i$ ) and $i i$ ) of Proposition 2.1.

Definition 2.3. (dual to Definition 2.2) We say that a quiver $Q$ is a sink projective representation quiver if for any $R$ the projective representation $\mathcal{X}$ of $(Q, R$-Mod) can be characterized in terms of the dual of the conditions $i$ ) and $i i$ ) of Proposition 2.1, that is,

i') For each vertex $v \in V$, the module $\mathcal{X}(v)$ is projective.

ii') For a vertex $v \in V$, the morphism $\oplus_{t(a)=v} \mathcal{X}(s(a)) \rightarrow \mathcal{X}(v)$ (where $\mathcal{X}(s(a)) \rightarrow \mathcal{X}(v)$ is $\mathcal{X}(a))$ is a splitting monomorphism.

We will denote by $\mathfrak{P}$ the subclass of the class of all sink projective representation quivers.

Left rooted quivers are examples of quivers in $\mathfrak{P}$, but as it is shown in $[2$, Theorem 4.1$], A_{\infty}^{\infty}$ is not a sink projective representation quiver. Cyclic quivers are also examples of quivers which do not lie in $\mathfrak{P}$.

\section{IndECOMPosable INJECTIVE REPRESENTATIONS.}

The main aim of this section is to characterize the indecomposable injective representations of trees. So let $T$ be a tree quiver. We recall that for a given $v$ as above, $e_{*}$ is the right adjoint of the restriction functor $(T, R$-Mod) $\rightarrow(\{v\}, R$-Mod) (where the last category is essentially $R$-Mod). 
Since our quiver is a tree, these representations have an especially simple form. These are such that $e_{*}^{v}(E)(w)=E$ if there is a path (necessarily unique) from $w$ to $v$ and such that $e_{*}^{v}(E)(w)=0$ otherwise. And $e_{*}^{v}(E)(a)=i d_{E}$ for any arrow $a$ such that $e_{*}^{v}(E)(t(a))=e_{*}^{v}(E)(s(a))=E$. This follows from Theorem 4.1 of [7]. It is easy to see that if $E$ is an indecomposable injective module then each $e_{*}^{v}(E)$ is also an indecomposable injective object of $(T, R$-Mod). But in general these $e_{*}^{v}(E)$ do not give all such objects. However, if we introduce the notion of vertices $w$ at infinity of $T$ and modify the construction of $e_{*}^{v}(E)$ to include objects $e_{*}^{w}(E)$ for such vertices at infinity, we will get all the indecomposable injective objects.

We begin by noting that given the vertex $v$ of $T$ there is a unique path $p=a_{n} a_{n-1} \cdots a_{1}$ of $Q$ such that $s\left(a_{1}\right)$ is the root of $T$ and such that $t\left(a_{n}\right)=v$. Then $e_{*}^{v}(E)$ is such that $e_{*}^{v}(E)\left(a_{i}\right)=i d_{E}$ for $i=1,2, \cdots, n$ and such that $e_{*}^{v}\left(v^{\prime}\right)=0$ when $v^{\prime}$ is none of $s\left(a_{1}\right), t\left(a_{1}\right), \cdots, s\left(a_{n}\right), t\left(a_{n}\right)$. We consider infinite paths $p=\cdots a_{3} a_{2} a_{1}$ such that $s\left(a_{1}\right)$ is the root of $T$. For each such path we associate a $w$ (distinct from all the vertices of $T$ ) that we call a vertex at infinity. With two distinct such paths we associate distinct vertices at infinity (we note the analogy with the procedure of adding points at infinity to form a projective space from an affine space).

Now let $p=\cdots a_{3} a_{2} a_{1}$ be an infinite path with $s\left(a_{1}\right)$ the root of the tree and with $w$ its associated vertex at $\infty$. For an injective left $R$-module $E$ we define the object $e_{*}^{w}(E)$ of $\left(T, R\right.$-Mod) to be that unique object such that $e_{*}^{w}(E)\left(a_{i}\right)=i d_{E}$ for each $a_{1}, a_{2}, a_{3}, \cdots$ and such that $e_{*}^{w}(E)(v)=0$ if $v$ is any vertex distinct from all the vertices $s\left(a_{j}\right), t\left(a_{j}\right)$ for $j=1,2,3, \cdots$.

We now shall prove

Proposition 3.1. If $w$ is a vertex at infinity for $T$ and if $E$ is an injective left $R$-module then $e_{*}^{w}(E)$ is an injective object of $(T, R-M o d)$. 
Proof. We first prove the claim when our tree is

$$
A_{\infty}^{+} \equiv \bullet \rightarrow \bullet \rightarrow \bullet \rightarrow \cdots
$$

(the infinite line to the right). Note that here we have one vertex at infinity, say $w$, and that then for $E, e_{*}^{w}(E)$ is just

$$
E \stackrel{i d}{\rightarrow} E \stackrel{i d}{\rightarrow} E \stackrel{i d}{\rightarrow} E \rightarrow \cdots
$$

Then note that if

$$
\mathcal{M}=M_{0} \rightarrow M_{1} \rightarrow M_{2} \rightarrow \cdots
$$

is any object of $\left(A_{\infty}^{+}, R\right.$-Mod $)$ then the morphisms $M \rightarrow e_{*}^{w}(E)$ are in one-to-one correspondence with maps $\lim _{\rightarrow} M_{n} \rightarrow E$. So then if $\mathcal{S}=S_{0} \rightarrow S_{1} \rightarrow S_{2} \rightarrow \cdots$ is a subobject of $M$, any morphism $S \rightarrow e_{*}^{w}(E)$ gives $\lim _{\rightarrow} S_{n} \rightarrow E$. But $E$ is injective and $\lim _{\rightarrow}$ is left exact, so $\lim _{\rightarrow} S_{n} \rightarrow E$ can be extended to $\lim _{\rightarrow} M_{n} \rightarrow E$. This in turn gives an extension $M \rightarrow e_{*}^{w}(E)$ and shows that $e_{*}^{w}(E)$ is injective.

Now we consider an arbitrary tree $T$. Given a vertex $w$ at infinity of $T$ there is an embedding $A_{\infty}^{+} \subseteq T$ that identifies $A_{\infty}^{+}$with the infinite path $p$ from the root of $T$ and having $w$ as its vertex at infinity. We now apply the comment at the bottom of page 302 of [7]. Letting $\mathcal{X}$ be our $e_{*}^{w}(E)$ (as an object in $\left(A_{\infty}^{+}, R\right.$-Mod)) and letting $f: A_{\infty}^{+} \rightarrow T$ be the embedding above we see that $f_{*}\left(e_{*}^{w}(E)\right)$ is precisely what we denote $e_{*}^{w}(E)$ as an object of $(T, R$-Mod). Since these right adjoint functors preserve injectivity (see Section 5, pg. 303 of [7]) we get that $e_{*}^{w}(E)$ is injective.

Note that if $E$ is also indecomposable then $e_{*}^{w}(E)$ is an indecomposable injective object of $(T, R$-Mod). 
Theorem 3.2. If $\mathcal{X}$ is an indecomposable injective object of $(T, R$-Mod) where $T$ is a tree, then $\mathcal{X} \cong e_{*}^{w}(E)$ for some vertex $w$ of $T$ (finite or infinite) and some indecomposable injective left $R$-module E.

Proof. We will use Proposition 2.1. Let $v_{0}$ be the root of $T$. If $\mathcal{X}\left(v_{0}\right) \rightarrow \prod_{s(a)=v_{0}} \mathcal{X}(t(a))$ is not an isomorphism, let $E$ be its kernel. Then $E$ is an injective left $R$-module. Also $e_{*}^{v_{0}}(E)$ is a nonzero injective subobject of $\mathcal{X}$. So since $\mathcal{X}$ is indecomposable, $\mathcal{X}=e_{*}^{v_{0}}(E)$.

So now suppose $\mathcal{X}\left(v_{0}\right) \rightarrow \prod_{s(a)=v_{0}} \mathcal{X}(t(a))$ is an isomorphism. For any arrow $b$ with $s(b)=v_{0}$ we then let $\mathcal{X}\left(v_{0}\right)=E^{\prime} \oplus E^{\prime \prime}$ corresponding to the decomposition $\prod \mathcal{X}(t(a))=\mathcal{X}(t(b)) \oplus$ $\prod_{a \neq b} \mathcal{X}(t(a))$. Consider the subobject $\mathcal{X}^{\prime}$ of $\mathcal{X}$ such that $\mathcal{X}^{\prime}\left(v_{0}\right)=E^{\prime}$ and then such that for $v \neq v_{0} \mathcal{X}(v)=0$ if the unique path from $v_{0}$ to $v$ does not go through $b$ and such that $\mathcal{X}^{\prime}(v)=\mathcal{X}(v)$ if it does.

Then let $\mathcal{X}^{\prime \prime}$ be the subobject of $\mathcal{X}$ such that $\mathcal{X}^{\prime \prime}\left(v_{0}\right)=E^{\prime \prime}$ and then such that for $v \neq v_{0}$, $\mathcal{X}^{\prime \prime}(v)=0$ if the path from $v_{0}$ to $v$ does go through $b$ and such that $\mathcal{X}^{\prime \prime}(v)=\mathcal{X}(v)$ otherwise. Checking the compatibility conditions we see that in fact $\mathcal{X}^{\prime}$ and $\mathcal{X}^{\prime \prime}$ are subobjects and then that $\mathcal{X}=\mathcal{X}^{\prime} \oplus \mathcal{X}^{\prime \prime}$. So by the indecomposability of $\mathcal{X}$ we get $\mathcal{X}=\mathcal{X}^{\prime}$ or $\mathcal{X}=\mathcal{X}^{\prime \prime}$.

Now since $\mathcal{X}\left(v_{0}\right) \rightarrow \prod \mathcal{X}(t(a))$ is an isomorphism and since $\mathcal{X}\left(v_{0}\right) \neq 0$ there is at least one $b$ such hat $\mathcal{X}(t(b)) \neq 0$. Choosing this $b$ we then get that $\mathcal{X}^{\prime} \neq 0$ and so that $\mathcal{X}=\mathcal{X}^{\prime}$.

So we note that our argument shows that for exactly one arrow $b$ with $s(b)=v_{0}$ we get that $\mathcal{X}(b)$ is an isomorphism and that $\mathcal{X}(a)=0$ for all $a \neq b$ with $s(a)=v_{0}$. Let $t(b)=v_{1}$ for this $b$. Now consider the subtree $T^{\prime} \subset T$ of $T$ with root $v_{1}$ and which contains all paths of $T$ beginning at $v_{1}$. If we repeat the arguments above with $T^{\prime}$ instead of $T$ we see that if we are then in the first situation we get $\mathcal{X}=e_{*}^{v_{1}}\left(\mathcal{X}\left(v_{0}\right)\right)$. If this does not occur then the procedure above will give 
us a $v_{2}$ and a corresponding subtree $T^{\prime \prime} \subset T$. Continuing we see that either the procedure stops and we get that $\mathcal{X}=e_{*}^{v_{n}}\left(\mathcal{X}\left(v_{0}\right)\right)$ for some vertex $v_{n}$. If the procedure does not stop then the vertices $v_{0}, v_{1}, v_{2}, \cdots$ will determine an infinite path beginning at $v_{0}$ and so a vertex $w$ at infinity such that $\mathcal{X}=e_{*}^{w}\left(\mathcal{X}\left(v_{0}\right)\right)$.

Remark. The $E$ and $w$ such that $\mathcal{X} \cong e_{*}^{w}(E)$ are uniquely determined up to isomorphisms.

\section{InJECTIVE REPRESENTATIONS OF RIGHT ROOTED QUIVERS}

In this section we prove that a wide class of quivers, the so called right rooted quivers, is contained in the class $\mathfrak{I}$ of all source injective representation quivers.

Right rooted quivers can be intuitively defined as follows:

Definition 4.1. 9, Proposition 3.6] A quiver $Q$ is right rooted (resp. left rooted quiver) if and only if there exists no path of the form $\bullet \rightarrow \bullet \rightarrow \bullet \rightarrow \cdots$ in $Q$ (resp. there exists no path of the form $\cdots \rightarrow \bullet \rightarrow \bullet \rightarrow \bullet$ in $Q)$.

We use the same notation as that introduced in [9] to characterize right rooted quivers in terms of certain subsets of the set of vertices, that is, for a quiver $Q=(V, \Gamma)$ we shall define by transfinite induction the following subsets of $V$,

$$
V_{0}=\{v \in V ; \text { there exists no arrow } a \text { of } Q \text { with } s(a)=v\}
$$

For a successor ordinal $\alpha$, we define

$$
V_{\alpha}=\left\{v \in V^{\alpha-1} ; \text { there exists no arrow } a \text { of } Q^{\alpha-1} \text { with } s(a)=v\right\} \text {, }
$$

where $Q^{\alpha-1}=\left(V^{\alpha-1}, \Gamma^{\alpha-1}\right)$ is the subquiver of $Q$ with

$$
V^{\alpha-1}=V \backslash V_{\alpha-1}
$$


and

$$
\Gamma^{\alpha-1}=\Gamma \backslash\left\{a \in \Gamma ; t(a) \in V_{\alpha-1}\right\} .
$$

For a limit ordinal we define

$$
V_{\omega}=\lim _{\rightarrow} V_{\alpha}=\cup_{\alpha<\omega} V_{\alpha}
$$

and $Q^{\omega}=\left(V^{\omega}, \Gamma^{\omega}\right)$ the subquiver of $Q$, where $V^{\omega}=V \backslash \cup_{\alpha<\omega} V_{\alpha}$ and

$$
\Gamma^{\omega}=\Gamma \backslash\left\{a \in \Gamma ; t(a) \in \lim _{\rightarrow} V_{\alpha}\right\}
$$

Then if a quiver is right rooted there will exist an ordinal number $\lambda$ such that $V=\cup_{\alpha<\lambda} V_{\alpha}$. The converse is also true by [9, Proposition 3.6].

Theorem 4.2. Let $Q$ be a right rooted quiver. Then $Q$ is a source injective representation quiver.

Proof. Let us see that conditions $i$ ) and $i$ ) of Proposition 2.1 are sufficient to get an injective representation. By transfinite induction on the set of vertices $V_{\alpha}$, we shall construct a family of injective subrepresentations of $\mathcal{E}$ whose direct product coincides with $\mathcal{E}$. Let $\mathcal{E}_{0}=$ $\prod_{v \in V_{0}} e_{*}^{v}(\mathcal{E}(v))$. Now, if $\alpha$ is a successor ordinal and $v \in V_{\alpha+1}$ it follows, by hypothesis,

$$
\mathcal{E}(v)=\left(\prod_{s(a)=v} \mathcal{E}(t(a))\right) \oplus E_{v}^{\alpha+1}
$$

(where the product $\prod_{s(a)=v} \mathcal{E}(t(a))$ is actually the product of $\mathcal{E}_{\mu}(v)$ with $\left.\mu \leq \alpha\right)$. Then we define

$$
\mathcal{E}_{\alpha+1}=\prod_{v \in V_{\alpha+1}} e_{*}^{v}\left(E_{v}^{\alpha+1}\right)
$$

Notice that $\mathcal{E}_{\alpha+1}(v)=0$ for all $v \in V_{\mu}, \mu<\alpha+1$. Now if $\gamma$ is a limit ordinal, we define

$$
\mathcal{E}_{\gamma}=\prod_{\alpha<\gamma} \mathcal{E}_{\alpha}
$$


Since the quiver is right rooted it is easy to see that there will exist an ordinal number $\lambda$ such that

$$
\mathcal{E}=\prod_{\alpha<\lambda} \mathcal{E}_{\alpha}
$$

and, since every $\mathcal{E}_{\alpha}$ is injective ( $e_{*}$ preserves injective objects), it follows that $\mathcal{E}$ will be also injective.

Example 1. Consider the quiver $T \equiv \bullet \rightarrow \bullet$, and the representations $\mathcal{S}=S_{1} \stackrel{\beta}{\rightarrow} S_{0}, \mathcal{X}=\mathcal{X}_{1} \stackrel{\alpha}{\rightarrow}$ $X_{0}$. If

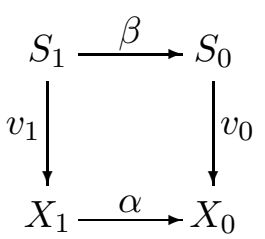

is a monomorphism of representations and

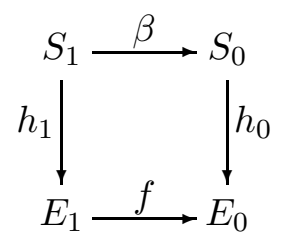

a morphism of representations with $E_{1} \stackrel{f}{\rightarrow} E_{0}$ an injective representation, we shall explicitly describe a morphism of representations $\left(t_{1}, t_{0}\right)$ extending $\left(h_{1}, h_{0}\right)$ via $\left(v_{1}, v_{0}\right)$. Since $E_{0}$ is injective there exists $t_{0}: X_{0} \rightarrow E_{0}$ such that $t_{0} \circ v_{0}=h_{0}$. On the other hand, since $f$ splits, let $g$ be a section. Then it follows that $E_{1}=\operatorname{Ker} f \oplus \operatorname{Im} g$, so we may define a morphism $\gamma: X_{1} \rightarrow \operatorname{Ker} f \operatorname{such}$ that $\gamma \circ v_{1}=p_{\operatorname{Ker} f} \circ h_{1}$ (where $p_{\operatorname{Ker} f}$ denotes the canonical projection of $E_{1}$ over $\operatorname{Ker} f$ ). Now we define $t_{1}: X_{1} \rightarrow E_{1}$ as $t_{1}=\gamma+g \circ t_{0} \circ \alpha$. It is immediate that $f \circ t_{1}=t_{0} \circ \alpha$. Let us see that $t_{1} \circ v_{1}=h_{1}$, so let $s_{1} \in S_{1}$ and suppose $h_{1}\left(s_{1}\right)=a+b$, with $a \in \operatorname{Ker} f$ and $b \in \operatorname{Im} g$ (so $b=g(x)$ 
for some $\left.x \in E_{0}\right)$. Then $\left(t_{1} \circ v_{1}\right)\left(s_{1}\right)=\left(\gamma v_{1}\right)\left(s_{1}\right)+\left(g t_{0} \alpha v_{1}\right)\left(s_{1}\right)=p_{\text {Kerf }}\left(h_{1}\left(s_{1}\right)\right)+\left(g t_{0} v_{0} \beta\right)\left(s_{1}\right)=$ $a+\left(g h_{0} \beta\right)\left(s_{1}\right)=a+\left(g f h_{1}\right)\left(s_{1}\right)=a+(g f)(a+b)=a+(g f)(g(x))=a+g(x)=a+b=h_{1}\left(s_{1}\right)$.

Example 2. The way of constructing extensions of the previous example can be easily generalized to many other right rooted quivers. For example,
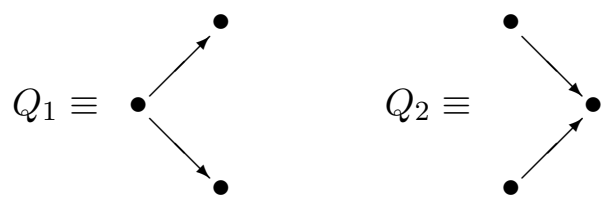

$Q_{3} \equiv \bullet \Longrightarrow$ or more generally to any dual tree or finite multiple (dual) tree. By the dual tree of a given one we mean a tree whose arrows are inverted, that is, a quiver with a vertex $v_{0}$ such that for any other vertex $w$ there exists a unique path from $w$ to $v_{0}$. A multiple (dual) tree is defined as a (dual) tree that admits multiple arrows from a vertex another (so it is not properly a (dual) tree).

Example 3. We now give an example of a non right rooted quiver such that conditions $i$ ) and ii) of Theorem 4.2 do not imply that a representation is injective. Let us consider the quiver

$$
Q \equiv \bigcirc
$$

and the category of representations of $Q$ over $k$-vector spaces, $(Q, k$-Mod) ( $k$ is a field). It is easy to see that $(Q, k$-Mod) is equivalent to the category $k[x]$-Mod. So now let us consider the representation with $k\left[x, x^{-1}\right]$ in the vertex and the morphism $k\left[x, x^{-1}\right] \stackrel{\cdot x}{\longrightarrow} k\left[x, x^{-1}\right]$. It is obvious that it satisfies $i$ ) and $i$ ) of Theorem 4.2, but $k\left[x, x^{-1}\right]$ is not divisible as a $k[x]$-module (in fact is $x$-divisible) so can not be injective. 


\section{InJeCtive REPRESEntations OF $A_{\infty}^{+}$}

In this section we develop new techniques (necessarily very different from those of Section 4) to characterize injective representations of non rooted right quivers. We focus our attention on the quiver $A_{\infty}^{+}$and then generalize this argument to any quiver such that the connected components are barren trees (so a forest of barren trees). Barren trees are important because they appear naturally in the study the existence of injective covers in $(Q, R$-Mod) (see [6, Theorem 3.6]) and also appear in the study of Gorenstein path rings, that is path rings which are left and right noetherian and whose projective objects have finite injective dimension (see [13]). We recall from [6] the definition of a barren tree: if $T$ is a tree with root $v$, we can divide the set of vertices into "states" in such a way that the first state $V_{1}=\{v\}$ and, for $i \in \mathbb{N}$,

$$
V_{i+1}=\left\{w \in V: \text { there exists } a: v_{i} \rightarrow w, \quad v_{i} \in V_{i}\right\}
$$

Then $T$ is barren if the sequence $\left(n_{i}\right)_{i \in \mathbb{N}}$ stabilizes (where $n_{i}=\left|V_{i}\right|$ ).

Example. The following is an example of an infinite barren tree.

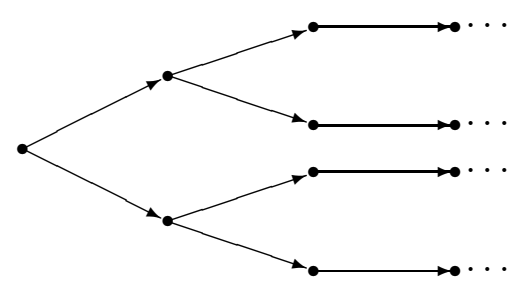

Let $\left(N_{i}\right)_{i \geq 0}$ be any family of modules. Let $U_{n}=\prod_{i=n}^{\infty} N_{i}$ and let $\mathcal{U}=U_{0} \rightarrow U_{1} \rightarrow \cdots$ where each $U_{n} \rightarrow U_{n+1}$ is the projection map. Then given $\mathcal{M}=M_{0} \stackrel{f_{0}}{\rightarrow} M_{1} \stackrel{f_{1}}{\rightarrow} \cdots$ and linear maps $\sigma_{n}: M_{n} \rightarrow N_{n}$ for each $n \geq 0$ there is a unique corresponding $\mathcal{M} \rightarrow \mathcal{N}$ such that $M_{n} \rightarrow U_{n}=\prod_{i=n}^{\infty} N_{i}$ is such that the composition $M_{n} \rightarrow \prod_{i=n}^{\infty} N_{i} \rightarrow N_{m}$ for $m \geq n$ is $\sigma_{n}$ if $m=n$ and is $\sigma_{m} \circ f_{m-1} \circ \cdots \circ f_{n}$ if $m>n$. And in fact every morphism $\mathcal{M} \rightarrow \mathcal{N}$ is of 
this form. So it is easy to see that if each $N_{i}$ is injective then $\mathcal{U}$ is injective. Also by choosing the $\sigma$ 's injective we can get an embedding into an injective representation. So every injective representation is a direct summand of such a $\mathcal{U}$.

Hence we consider direct sum decompositions of arbitrary representations $\mathcal{M}$, say $\mathcal{M}=\mathcal{M}^{\prime} \oplus \mathcal{M}^{\prime \prime}$ where $\mathcal{M}=M_{0} \rightarrow \cdots$. Such a decomposition is given by decompositions $M_{n}=M_{n}^{\prime} \oplus M_{n}^{\prime \prime}$ for each $n$, but which are compatible with the maps $M_{n} \rightarrow M_{n+1}$, i.e. which map $M_{n}^{\prime}$ into $M_{n+1}^{\prime}$ and $M_{n}^{\prime \prime}$ into $M_{n+1}^{\prime \prime}$. So this just says $M_{n} \rightarrow M_{n+1}$ is homogeneous with respect to the decompositions. Given such a decomposition we easily see that each $\operatorname{Ker}\left(M_{n} \rightarrow M_{m}\right)(m \geq n)$ is a homogeneous submodule (again with respect to the decomposition). This means that $K=\left(K \cap M_{n}^{\prime}\right) \oplus\left(K \cap M_{n}^{\prime \prime}\right)$ where $K$ is this kernel.

If now in fact $\mathcal{M}$ is such that each $M_{n} \rightarrow M_{n+1}$ is surjective then letting $\mathcal{M}=M \rightarrow M / K_{0} \rightarrow$ $M / K_{1} \cdots$ with $K_{0} \subset K_{1} \cdots$ it is not hard to see that to get a decomposition of $\mathcal{M}$ we only need give a direct sum decomposition $M=M^{\prime} \oplus M^{\prime \prime}$ such that each of the $K_{i}$ is a homogeneous submodule. Clearly this remark applies to any $M_{0} \rightarrow M_{1} \rightarrow \cdots$ where all the maps are surjective. This means that to give a decomposition of this representation we only need give one of $M_{0}$ such that all the kernels $M_{0} \rightarrow M_{n}$ are homogeneous for every $n \geq 1$.

We now apply these remarks.

Theorem 5.1. Let $\left(E_{i}\right)_{i \geq 0}$ be a family of injective modules. Let $\oplus_{i=0}^{\infty} E_{i} \subset E$ be an injective envelope. Then the representation

$$
\overline{\mathcal{E}}=E \rightarrow E / E_{0} \rightarrow E /\left(E_{0} \oplus E_{1}\right) \rightarrow \cdots
$$


is an injective envelope of

$$
\mathcal{E}=\oplus_{i=0}^{\infty} E_{i} \rightarrow \oplus_{i=1}^{\infty} E_{i} \rightarrow \cdots
$$

Proof. We use the earlier remarks to construct a known injective representation which is isomorphic to $\overline{\mathcal{E}}$. We let $\mathcal{U}$ be the representation $\mathcal{U}=\prod_{i=0}^{\infty} E_{i} \rightarrow \prod_{i=1}^{\infty} E_{i} \rightarrow \cdots$. This representation is injective. We will try to find a direct summand which is isomorphic to the representation of the theorem. So we construct a direct sum decomposition of $\mathcal{U}$. This representation is such that the maps $\prod_{i=n}^{\infty} E_{i} \rightarrow \prod_{i=n+1}^{\infty} E_{i}$ are all surjective. So we use this fact to construct our decomposition. Let $\oplus_{i=0}^{\infty} E_{i} \subset E^{\prime} \subset \prod_{i=0}^{\infty} E_{i}$ where $E^{\prime}$ is an injective envelope of $\oplus_{i=0}^{\infty} E_{i}$.

Since $E^{\prime}$ is injective we have a decomposition $\prod_{i=0}^{\infty} E_{i}=E^{\prime} \oplus E^{\prime \prime}$. We claim $\operatorname{ker}\left(\prod_{i=0}^{\infty} E_{i} \rightarrow\right.$ $\left.\prod_{i=n}^{\infty} E_{i}\right)=E_{0} \oplus \cdots \oplus E_{n-1}$ is homogeneous with respect to this decomposition. This is trivial since $E_{0} \oplus \cdots E_{n-1} \subset E^{\prime}$ for each $n \geq 1$. So we now use our procedure and construct the corresponding decomposition $\mathcal{E}=\mathcal{E}^{\prime} \oplus \mathcal{E}^{\prime \prime}$. By construction it is clear that $\mathcal{E}^{\prime}$ is isomorphic to the representation $\overline{\mathcal{E}}=E \rightarrow E / E_{0} \rightarrow E /\left(E_{0} \oplus E_{1}\right) \rightarrow \cdots$. Hence this representation is injective. To see that it is an envelope, note that $E /\left(E_{0} \oplus \cdots \oplus E_{n-1}\right)$ is an injective envelope of $\oplus_{i=n}^{\infty} E_{i}$ for each $n$. Hence any $\overline{\mathcal{E}} \rightarrow \overline{\mathcal{E}}$ that makes the obvious diagram commutative gives automorphisms of each $E /\left(E_{0} \oplus \cdots \oplus E_{n-1}\right)$ and so $\mathcal{E} \rightarrow \mathcal{E}$ is an automorphism.

We now want to give a characterization of injective representations. We will need

Lemma 5.2. If $E$ is an injective module then $\mathcal{E}=E \rightarrow E \rightarrow \cdots$ is an injective representation.

Proof. This follows from the observation (made in the first part of the proof of Proposition 3.1) that morphisms $\mathcal{M} \rightarrow \mathcal{E}$ are given by maps $\lim _{\rightarrow} M_{n} \rightarrow E$. 
So we note that any $\mathcal{E}=E_{0} \rightarrow E_{1} \rightarrow \cdots$ with all $E_{n}$ injective and each $E_{n} \rightarrow E_{n+1}$ an isomorphism is an injective representation.

We note that there is a natural torsion theory on the category of representations where $\mathcal{M}=$ $M_{0} \rightarrow \cdots$ is torsion if for each $n$ and each $x \in M_{n}$ there is an $m>n$ such that $x \in \operatorname{ker}\left(M_{n} \rightarrow\right.$ $\left.M_{m}\right)$. Then for an arbitrary representation $\mathcal{M}$ we let $t(\mathcal{M})$ be the torsion subrepresentation. Note that $N_{0} \rightarrow N_{1} \rightarrow \cdots$ torsion free just means that each $N_{n} \rightarrow N_{n+1}$ is an injection.

Theorem 5.3. A representation $\mathcal{G}=G_{0} \rightarrow \cdots$ is injective if and only if each $G_{n}$ is injective and each $G_{n} \rightarrow G_{n+1}$ is surjective with an injective kernel, that is, $A_{\infty}^{+} \in \mathfrak{I}$.

Proof. By Proposition 2.1 we know the conditions are necessary, so assume these conditions. We want to argue that $\mathcal{G}$ is injective. Let $E_{0}=\operatorname{ker}\left(G_{0} \rightarrow G_{1}\right)$. Then $E_{0}$ is injective and is a submodule of the injective $\operatorname{Ker}\left(G_{0} \rightarrow G_{2}\right)$. So we can find an $E_{1}$ so that $E_{0} \oplus E_{1}=\operatorname{Ker}\left(G_{0} \rightarrow\right.$ $\left.G_{2}\right)$. We proceed in this manner and find $E_{n}$ for all $n$ so that $\oplus_{i=0}^{n-1} E_{i}=\operatorname{Ker}\left(G_{0} \rightarrow G_{n}\right)$. Let $\oplus_{i=0}^{\infty} E_{i} \subset E \subset G_{0}$ where $E$ is an injective envelope of $\oplus_{i=0}^{\infty} E_{i}$. Then up to isomorphism, $\overline{\mathcal{E}}=E \rightarrow E / E_{0} \rightarrow E /\left(E_{0} \oplus E_{1}\right) \rightarrow \cdots$ is a subrepresentation of $\mathcal{G}$. By the above it is an injective representation and in fact is the injective envelope of $\mathcal{E}=\oplus_{i=0}^{\infty} E_{i} \rightarrow \oplus_{i=1}^{\infty} E_{i} \rightarrow \cdots$. Clearly $\mathcal{E}$ is $t(\mathcal{G})$. So $\mathcal{G}=\overline{\mathcal{E}} \oplus \mathcal{G}^{\prime}$. Since $\overline{\mathcal{E}} \supset t(\mathcal{G}), \mathcal{G}^{\prime}$ is torsion free. But then $\mathcal{G}^{\prime}$ has all its terms injective and all its maps surjective. But this means all its maps are isomorphisms. Hence $\mathcal{G}^{\prime}$ is injective and so $\mathcal{G}$ as the direct sum of two injectives is injective.

Remark 1. From the arguments it is easy to see that an injective representation is uniquely determined up to isomorphism by the family $\left(E_{i}\right)_{i \geq 0}$ as above and the single injective module 
$E$ so that the torsion free quotient of the injective is isomorphic to $E \rightarrow E \rightarrow \cdots$.

Remark 2. Since a torsion theory is stable if and only if injective envelopes of torsion objects are torsion we see that if our theory is stable, then using the notation above, $\oplus_{i=0}^{\infty} E_{i}$ must be its own injective envelope for any family $\left(E_{i}\right)_{i \geq 0}$ of injective modules. So this means the ring $R$ must be left noetherian. If conversely $R$ is left noetherian then by our arguments we see that for any injective $\mathcal{G}, t(\mathcal{G})$ is also injective. This quickly gives that injective envelopes of torsion objects are also torsion. So the torsion theory is stable. Hence we get that $R$ is left noetherian if and only if our torsion theory is stable.

Theorem 5.3 allows us to prove that some non right rooted quivers are also source injective representation quivers.

Corollary 5.4. Let $\mathcal{E}=\cdots \rightarrow E_{-2} \rightarrow E_{-1} \rightarrow E_{0} \rightarrow E_{1} \rightarrow E_{2} \rightarrow \cdots$ be a representation of the quiver

$$
A_{\infty}^{\infty} \equiv \cdots \rightarrow \bullet \rightarrow \bullet \rightarrow \cdots
$$

Then $\mathcal{E}$ is injective if, and only if, $E_{i}$ is an injective $R$-module and $E_{i} \rightarrow E_{i+1}$ is an splitting epimorphism for all $i \in \mathbb{Z}$, that is, the quiver $A_{\infty}^{\infty}$ lies in the class $\mathfrak{I}$.

Proof. Let $0 \rightarrow \mathcal{S} \stackrel{g}{\rightarrow} \mathcal{X}$ and $\mathcal{S} \stackrel{h}{\rightarrow} \mathcal{E}$ be a morphism of representations of $A_{\infty}$ (so $g=\left(g_{i}\right)_{i \in \mathbb{Z}}$ and $\left.h=\left(h_{i}\right)_{i \in \mathbb{Z}}\right)$. By Theorem 5.3 there exist morphisms $\left(t_{i}\right)_{i \geq 0}$ such that $\left(h_{i}\right)=\left(t_{i}\right) \circ\left(g_{i}\right)$ and $\left(t_{i}\right)$ satisfying the usual compatibility conditions, for all $i \geq 0$. Now from $t_{0}: X_{0} \rightarrow E_{0}$ we get, by Example 1, a morphism $t_{-1}: X_{-1} \rightarrow E_{-1}$ such that $\left(t_{-1}, t_{0}\right)$ extends $\left(h_{-1}, h_{0}\right)$ and verifies 
the compatibility condition. Now proceed inductively to get a family $t_{-i}: X_{-i} \rightarrow E_{-i}$, for all $i \geq 1$ by applying Example 1 iteratively.

We finish by characterizing injective representations for infinite barren trees.

Corollary 5.5. Let $Q$ be a forest whose connected components are barren trees. Then $Q \in \mathfrak{I}$.

Proof. We need to check that conditions $i$ ) and $i$ ) of Proposition 2.1 are sufficient to get an injective representation.

It is clear that we only need to prove the result for a barren tree $T$. Since $T$ is barren there exists $k \in \mathbb{N}$ such that $n_{k}=n_{k+i}$, for all $i \geq 0$. Now suppose that $V_{k}=\left\{v_{1}, \cdots, v_{m}, v_{m+1}, \cdots, v_{n_{k}}\right\}$, where we denote by $v_{1}, \cdots, v_{m}$ the vertices at infinity, so we have

$$
v_{j} \equiv v_{j}^{0} \rightarrow v_{j}^{1} \rightarrow v_{j}^{2} \cdots
$$

for $1 \leq j \leq m$ and suppose (without loss of generality) that $v_{j}^{0} \in V_{k}$, for all $1 \leq j \leq m$.

Now let $\mathcal{E}$ be a representation of $T$ satisfying $i$ ) and $i i)$ of Proposition 2.1 and $0 \rightarrow \mathcal{S} \stackrel{g}{\rightarrow} \mathcal{X}$, $\mathcal{S} \stackrel{h}{\rightarrow} \mathcal{E}$ two morphisms of representations over $T$. Let us consider the corresponding restrictions $g\left(v_{j}\right)$ and $h\left(v_{j}\right)$ to the vertices at infinity of $V_{k}, 1 \leq j \leq m$. Then by Theorem 5.3 , for each $1 \leq j \leq m$ there exists a family of morphisms

$$
t\left(v_{j}\right)=\left\{t\left(v_{j}^{i}\right): \quad i \in \mathbb{N}\right\}
$$

such that $t\left(v_{j}^{i}\right) \circ g\left(v_{j}^{i}\right)=h\left(v_{j}^{i}\right)$, for all $1 \leq j \leq m, i \in \mathbb{N}$ and for a fixed $1 \leq j \leq m$ the morphisms $\left\{t\left(v_{j}^{i}\right): \quad i \in \mathbb{N}\right\}$ induce a morphism of representations over the quiver $v_{j} \equiv v_{j}^{0} \rightarrow v_{j}^{1} \rightarrow v_{j}^{2} \cdots$

Now from the morphisms

$$
\left\{t\left(v_{j}^{0}\right): \quad 1 \leq j \leq m\right\} \cup\left\{t\left(v_{j}\right): m \leq j \leq n_{k}\right\}
$$


related to vertices of $V_{k}$, we may construct the corresponding extensions to vertices of $V_{k-1}$ as it is shown in Example1(and by the comments made in Example 2) and following repeatedly until we reach to the root $V_{1}=\{v\}$. So at the end we will have a representation $t: \mathcal{X} \rightarrow \mathcal{E}$ extending $h: \mathcal{S} \rightarrow \mathcal{E}$ via $g: \mathcal{S} \rightarrow \mathcal{X}$ and satisfying the compatibility condition, that is, if $a: v \rightarrow w$ is an arrow of $T$ the diagram

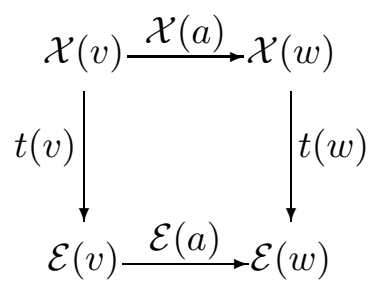

is commutative.

Remark. If the ring $0 \neq R$ is left noetherian and again our quiver is a tree $T$, then in [6] it was proved that $(T, R$-Mod) is a locally noetherian Grothendieck category if and only if $T$ is barren. In this case every injective object of $(T, R$-Mod) is uniquely up to isomorphism the direct sum of the indecomposable injectives $e_{*}^{w}(E)$ of Section 3 ,

\section{Applications: Gorenstein Representations}

As an application of the results of the previous sections, we study and characterize the representations of finite injective dimension (resp. finite projective dimension) and its right orthogonal class (resp. its left orthogonal class) with respect to the $\operatorname{Ext}^{1}$ functor, that is, the class of Gorenstein injective (resp. Gorenstein projective) representations. We also study Gorenstein flat representations and give and upper bound of the Gorenstein injective dimension of a representation having finite Gorenstein injective dimension on every module associated with each vertex. 
We recall (see, for example, [8]):

Definition 6.1. An object $M$ of an abelian category $\mathcal{A}$ is said to be Gorenstein injective if there is an exact sequence

$$
\cdots \rightarrow E_{-3} \rightarrow E_{-2} \rightarrow E_{-1} \rightarrow E_{0} \rightarrow E_{1} \rightarrow E_{2} \rightarrow \cdots
$$

of injective objects such that $M=\operatorname{Ker}\left(E_{0} \rightarrow E_{1}\right)$ and such that the sequence is $\operatorname{Hom}(E,-)$-exact for every injective object $E$ (i.e. the functor $\operatorname{Hom}(E,-)$ leaves the sequence exact).

We also recall from [4] the definition of a Gorenstein category.

Definition 6.2. We will say that a Grothendieck category $\mathcal{A}$ is a Gorenstein category if the following hold:

1) For any object $L$ of $\mathcal{A}, \operatorname{projdim} L<\infty$ if and only if $\operatorname{injdim} L<\infty$.

2) Finitistic projective dimension and finitistic injective dimension of $\mathcal{A}$ are both finite, that is,

$$
F P D(\mathcal{A})=\sup \{\operatorname{projdim}(M): \operatorname{projdim}(M)<\infty\}
$$

and

$$
F I D(\mathcal{A})=\sup \{\operatorname{inj} \operatorname{dim}(M): \operatorname{inj} \operatorname{dim}(M)<\infty\}
$$

3) $\mathcal{A}$ has a generator $L$ such that $\operatorname{projdim} L<\infty$.

Then in [5] it is proved that, for any arbitrary quiver $Q$, if $R$ is such that $R$-Mod is Gorenstein (for example if $R$ is an $n$-Gorenstein ring) then $(Q, R$-Mod) is a Gorenstein category and so by [4, Theorem 2.25] the pair of classes $\left(\mathfrak{L}, \mathfrak{L}^{\perp}\right)$ where $\mathfrak{L}$ is the class of representations of finite injective dimension and $\mathfrak{L}^{\perp}$ is the class of Gorenstein injective representations, is a complete cotorsion 
pair. Analogously, the pair $\left({ }^{\perp} \mathfrak{D}, \mathfrak{D}\right)$ where $\mathfrak{D}$ is the class of objects of finite projective dimension and ${ }^{\perp} \mathfrak{D}$ is the class of Gorenstein projective representations, is also a complete cotorsion pair.

We now characterize the representations of the classes $\mathfrak{L}$ and $\mathfrak{L}^{\perp}\left(\right.$ resp. ${ }^{\perp} \mathfrak{D}$ and $\left.\mathfrak{D}\right)$ for source injective representation quivers.

Corollary 6.3. Let $Q$ be a source injective representation quiver and $R$ be a Gorenstein ring (see [13]). A representation $\mathcal{M}$ of the quiver $Q$ is Gorenstein injective if and only if for each vertex $v \mathcal{M}(v)$ is a Gorenstein injective $R$-module and the sequence

$$
0 \rightarrow \operatorname{Ker}\left(f_{v}\right) \rightarrow \mathcal{M}(v) \stackrel{f_{v}}{\rightarrow} \prod_{s(a)=v} \mathcal{M}(t(a)) \rightarrow 0
$$

is exact with $\operatorname{Ker}\left(f_{v}\right)$ Gorenstein injective (where $f_{v}: \mathcal{M}(v) \rightarrow \prod_{s(a)=v} \mathcal{M}(t(a)$ ) is the induced by the $\mathcal{M}(a): \mathcal{M}(v) \rightarrow \mathcal{M}(t(a)))$.

Proof. Suppose $\mathcal{M}$ is Gorenstein injective, then there will exist an exact sequence of injectives

$$
\cdots \rightarrow \mathcal{E}_{-2} \rightarrow \mathcal{E}_{-1} \rightarrow \mathcal{E}_{0} \rightarrow \mathcal{E}_{1} \rightarrow \mathcal{E}_{2} \rightarrow \cdots
$$

with $\mathcal{M}=\operatorname{Ker}\left(\mathcal{E}_{0} \rightarrow \mathcal{E}_{1}\right)$, which is $\operatorname{Hom}(\mathcal{E},-)$ exact, for all injective representations. Then, for a fixed vertex $v$ we have the corresponding exact sequence of injective modules

$$
\cdots \rightarrow \mathcal{E}_{-2}(v) \rightarrow \mathcal{E}_{-1}(v) \rightarrow \mathcal{E}_{0}(v) \rightarrow \mathcal{E}_{1}(v) \rightarrow \mathcal{E}_{2}(v) \rightarrow \cdots
$$

with $\mathcal{M}(v)=\operatorname{Ker}\left(\mathcal{E}_{0}(v) \rightarrow \mathcal{E}_{1}(v)\right)$. Then, taking an integer sufficiently large in absolute value, and apply [8, Theorem 9.1.11(7)], we will have that $\mathcal{M}(v)$ is Gorenstein injective for all vertex $v$. Now, since $\mathcal{M}$ is Gorenstein injective, there will exist a short exact sequence of representations

$$
0 \rightarrow \mathcal{U} \rightarrow \mathcal{E} \rightarrow \mathcal{M} \rightarrow 0
$$


with $\mathcal{E}$ injective and $\mathcal{U}$ Gorenstein injective. By Proposition 2.1, $\mathcal{E}(v) \rightarrow \prod_{s(a)=v} \mathcal{E}(t(a))$ is a splitting epimorphism, so it easily follows that $\mathcal{M}(v) \rightarrow \prod_{s(a)=v} \mathcal{M}(t(a))$ is an epimorphism. Finally let us show that $\operatorname{Ker}\left(f_{v}\right)$ is Gorenstein injective. By Proposition 2.1 we have, from (6.1) the exact sequence of injective kernels:

$$
\cdots \rightarrow \operatorname{Ker}\left(t_{v}\right)^{-2} \rightarrow \operatorname{Ker}\left(t_{v}\right)^{-1} \rightarrow \operatorname{Ker}\left(t_{v}\right)^{0} \rightarrow \operatorname{Ker}\left(t_{v}\right)^{1} \rightarrow \operatorname{Ker}\left(t_{v}\right)^{2} \rightarrow \cdots
$$

where

$$
0 \rightarrow \operatorname{Ker}\left(t_{v}\right)^{j} \rightarrow E_{j}(v) \stackrel{t_{v}}{\longrightarrow} \prod_{s(a)=v} E_{j}(t(a)) \rightarrow 0
$$

for all $j \in \mathbb{Z}$, such that $\operatorname{Ker}\left(f_{v}\right)=\operatorname{Ker}\left(\operatorname{Ker}\left(t_{v}\right)^{0} \rightarrow \operatorname{Ker}\left(t_{v}\right)^{1}\right)$ which is $\operatorname{Hom}(E,-)$ exact for all injective $R$-modules $E$, by a reasoning similar to the preceding.

Let us see that the conditions are sufficient. Since $(Q, R$-Mod) is a Gorenstein category we may find a short exact sequence

$$
0 \rightarrow \mathcal{G} \rightarrow \mathcal{L} \rightarrow \mathcal{M} \rightarrow 0
$$

with $\mathcal{G}$ a Gorenstein injective representation and $\mathcal{L}$ a representation of finite injective dimension. By the previous proof $\mathcal{G}(v)$ is a Gorenstein injective $R$-module and $\operatorname{Ker}\left(h_{v}\right)$ in the exact

$$
0 \rightarrow \operatorname{Ker}\left(h_{v}\right) \rightarrow \mathcal{G}(v) \stackrel{h_{v}}{\longrightarrow} \prod_{s(a)=v} \mathcal{G}(t(a)) \rightarrow 0
$$

is also Gorenstein injective. Moreover, since $\mathcal{L}$ is of finite injective dimension, it is obvious that $\mathcal{L}(v)$ and the kernel of $\mathcal{L}(v) \rightarrow \prod_{s(a)=v} \mathcal{L}(t(a))$ is also of finite injective dimension (and this map is a surjection). So by the definition of a source injective representation quiver it follows that $\mathcal{L}$ is an injective representation, so $\mathcal{M}$ will be also Gorenstein injective.

In a dual manner and using the results of [2] we can characterize Gorenstein projective representations of quivers. 
Corollary 6.4. Let $Q$ be a sink projective representation quiver and let $R$ be a Gorenstein ring. A representation $\mathcal{M}$ of the quiver $Q$ is Gorenstein projective if and only if $\mathcal{M}(v)$ is a Gorenstein projective $R$-module, the sequence

$$
0 \rightarrow \oplus_{t(a)=v} \mathcal{M}(s(a)) \stackrel{f_{v}}{\longrightarrow} \mathcal{M}(v) \rightarrow \mathcal{C}(v) \rightarrow 0
$$

is exact and $\mathcal{C}(v)$ is Gorenstein projective (where $f_{v}: \oplus_{t(a)=v} \mathcal{M}(s(a)) \rightarrow \mathcal{M}(v)$ is the induced by $\mathcal{M}(s(a)) \rightarrow \mathcal{M}(v))$.

We can also characterize the class of all representations of finite injective dimension for quivers in $\mathfrak{I}$.

Proposition 6.5. A representation $\mathcal{M}$ of a source injective representation quiver is of finite injective dimension if and only if $\sup \left\{\operatorname{inj}_{\operatorname{dim}} \mathcal{M}(v)<\infty: v \in V\right\}$ is finite.

Proof. It is obvious that the condition is necessary, for if $\operatorname{inj}_{\operatorname{dim}} \mathcal{M} \leq n$ then, for all vertex $v \in V, \operatorname{injdim}_{R} \mathcal{M}(v) \leq n$

Conversely, suppose that $n=\sup \left\{\operatorname{inj}_{\operatorname{dim}} \mathcal{M}(v)<\infty: v \in V\right\}$, and let $v \in V$ be a vertex, then there exists an exact sequence of injective $R$-modules,

$$
0 \rightarrow \mathcal{M}(v) \rightarrow E_{0}^{v} \rightarrow E_{1}^{v} \rightarrow \cdots \rightarrow E_{n}^{v} \rightarrow 0
$$

But then we have the short exact sequence of representations

$$
0 \rightarrow \mathcal{M} \rightarrow \prod_{v \in V} e_{*}^{v}\left(E_{0}^{v}\right) \rightarrow \mathcal{M}_{1} \rightarrow 0
$$

such that $\operatorname{inj}_{\operatorname{dim}} \mathcal{M}_{1}(v) \leq n-1$, for every $v \in V$. Now we repeat the same procedure to get $\mathcal{M}_{2}$ from $\mathcal{M}_{1}$ satisfying $\operatorname{injdim}_{R} \mathcal{M}_{2}(v) \leq n-2$, for all $v \in V$. So finally we get the exact

$$
0 \rightarrow \mathcal{M} \rightarrow \mathcal{E}_{0} \rightarrow \mathcal{E}_{1} \rightarrow \cdots \rightarrow \mathcal{E}_{n-1} \rightarrow \mathcal{M}_{n} \rightarrow 0
$$


such that, $\mathcal{M}_{n}(v)$ is injective, for all vertex $v \in V$. Let us show that $\operatorname{injdim}_{Q} M \leq 1$. For if

$$
0 \rightarrow \mathcal{M}_{n} \rightarrow \mathcal{E}_{n} \rightarrow \mathcal{C} \rightarrow 0
$$

is an exact sequence with $\mathcal{E}_{n}$ injective, by Proposition 2.1, we have that $\mathcal{C}(v)$ is an injective $R$-module and $\mathcal{C}(v) \rightarrow \prod_{s(a)=v} \mathcal{C}(t(a))$ is an epimorphism with injective kernel, for all $v \in V$, so since the quiver is a source injective representation quiver, $\mathcal{C}$ will be an injective representation.

Remark. Notice that the previous proposition shows that if $\sup \left\{\operatorname{inj}_{\operatorname{dim}} \mathcal{M}(v)<\infty: v \in\right.$ $V\} \leq n$ then $\operatorname{inj}_{\operatorname{dim}} \mathcal{M} \leq n+1$. The converse clearly is not true.

When $(Q, R$-Mod) is a Gorenstein category, we can define the Gorenstein injective dimension of a representation $\mathcal{M}$ as the least natural number $k$ such that the $n$th cosyzygy of $\mathcal{M}$ is Gorenstein injective, that is, $k$ is the least integer such that there exists an exact sequence

$$
0 \rightarrow \mathcal{M} \rightarrow \mathcal{E}_{0} \rightarrow \mathcal{E}_{1} \rightarrow \cdots \rightarrow \mathcal{E}_{k-1} \rightarrow \mathcal{G}_{k} \rightarrow 0
$$

with $\mathcal{E}_{i}$ injective $0 \leq i \leq k-1$ and $\mathcal{G}_{k}$ Gorenstein injective $(k=\infty$ if there exists no such a natural number). We denote the Gorenstein injective dimension of a representation by $\operatorname{Ginjdim}_{Q} \mathcal{M}$.

Proposition 6.6. Let $(Q, R$-Mod) be a Gorenstein category and let $\mathcal{M}$ be a representation. Suppose that $\mathcal{M}(v)$ is a Gorenstein injective $R$-module for all $v \in V$, then Ginjdim $_{Q} \mathcal{M} \leq 1$.

Proof. We consider a short exact sequence $0 \rightarrow \mathcal{M} \rightarrow \mathcal{G} \rightarrow \mathcal{C} \rightarrow 0$ with $\mathcal{G}$ a Gorenstein injective representation and $\operatorname{inj}_{\operatorname{dim}} \mathcal{C}<\infty$. Let $\mathcal{E} \rightarrow \mathcal{G} \rightarrow 0$ be exact with $\mathcal{E}$ injective. We have the pullback diagram 


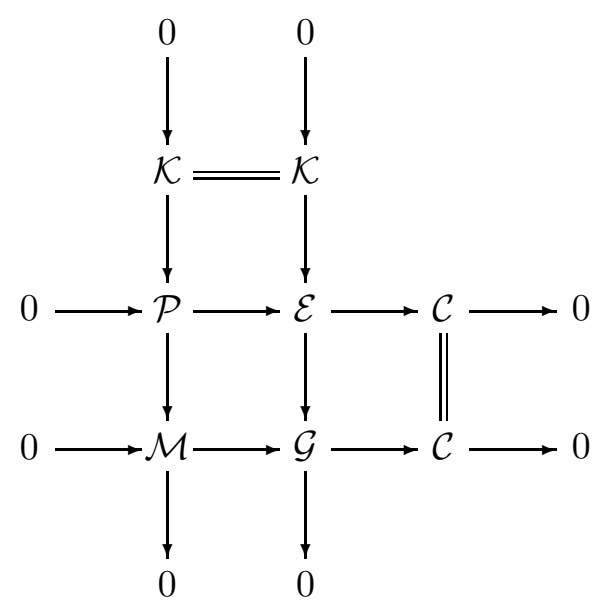

Now $\mathcal{K}$ is a Gorenstein injective representation, so by Corollary 6.3 and the hypothesis, we have that $\mathcal{P}(v)$ is a Gorenstein injective $R$-module. Since $\operatorname{inj}_{\operatorname{dim}} \mathcal{C}<\infty$ it follows that $\operatorname{inj} \operatorname{dim}_{Q} \mathcal{P}<$ $\infty$, so by Proposition 6.5 we conclude that $\mathcal{P}(v)$ is an injective $R$-module. But then the previous remark says that $\operatorname{inj}_{\operatorname{dim}} \mathcal{P} \leq 1$. Now we form the pushout diagram of $0 \rightarrow \mathcal{P} \rightarrow \mathcal{E}(\mathcal{P})$ (the injective hull) and $\mathcal{P} \rightarrow \mathcal{M} \rightarrow 0$

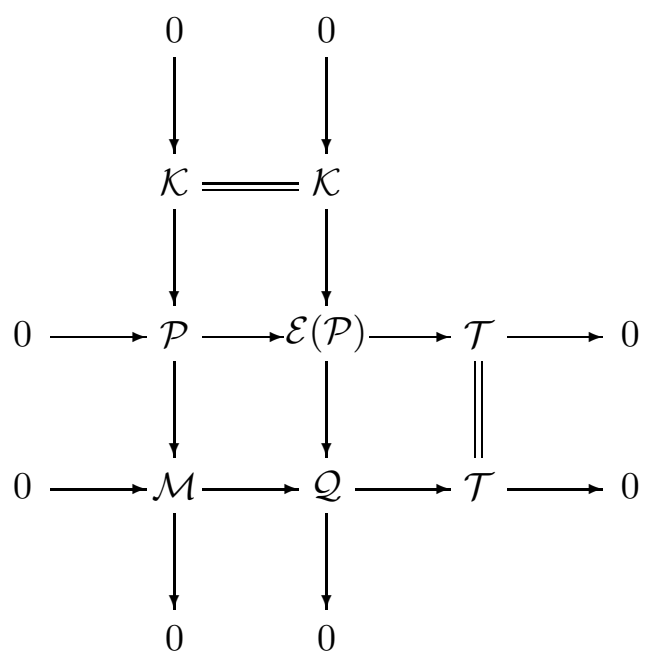

Since $\mathcal{K}$ is Gorenstein injective, $\mathcal{Q}$ will be Gorenstein injective and since $\operatorname{inj}_{\operatorname{dim}} \mathcal{P} \leq 1, \mathcal{T}$ will be injective, so $\operatorname{Ginjdim}_{Q} \mathcal{M} \leq 1$. 
Remark. The argument of the previous proposition can be easily extended and show that if $\operatorname{Ginjdim}_{Q} \mathcal{M}(v) \leq k$, for all $v \in V$ then $\operatorname{Ginjdim}_{Q} \mathcal{M} \leq k+1$.

In [9], the authors characterize flat representations of left rooted quivers. We may use this result with Theorem 4.2 to relate flat and injective representations. We need to introduce the following notation: we denote by $\left(Q^{o p}, R\right.$-Mod $)$ the category of representations over the quiver $Q^{o p}=\left(V, \Gamma^{o p}\right)$, with the same set of vertices as $Q$ and where the arrows are the arrows of $\Gamma$ reversed (so $v \rightarrow w \in \Gamma^{o p} \Leftrightarrow w \rightarrow v \in \Gamma$ ). It is obvious that if $Q$ is left rooted then $Q^{o p}$ is right rooted.

Corollary 6.7. Let $Q$ be a left rooted quiver. Then $\mathcal{F}$ is a flat representation over $(Q, R-M o d)$ if, and only if, $\mathcal{F}^{+}$is an injective representation over the quiver $\left(Q^{o p}\right.$, Mod-R) (where $\mathcal{F}^{+}$is the representation given by $\mathcal{F}^{+}(v)=\operatorname{Hom}_{\mathbb{Z}}(\mathcal{F}(v), \mathbb{Q} / \mathbb{Z})$ and, for an arrow $a: v \rightarrow w$ and the morphism $\left.f_{a}: \mathcal{F}(v) \rightarrow \mathcal{F}(w), f_{a^{o p}}^{+}=\operatorname{Hom}_{\mathbb{Z}}\left(f_{a}, \mathbb{Q} / \mathbb{Z}\right): \mathcal{F}^{+}(w) \rightarrow \mathcal{F}^{+}(v)\right)$

Proof. This follows from [9, Theorem 3.7] and Theorem 4.2 by noticing that

$$
0 \rightarrow \oplus_{t(a)=v} \mathcal{F}(s(a)) \stackrel{f_{v}}{\rightarrow} \mathcal{F}(v) \stackrel{p_{v}}{\rightarrow} \mathcal{C}(v) \rightarrow 0
$$

is a pure exact sequence of left $R$-modules if, and only if,

$$
0 \rightarrow \mathcal{C}^{+}(v) \stackrel{p_{v}^{+}}{\longrightarrow} \mathcal{F}^{+}(v) \stackrel{f_{v}^{+}}{\longrightarrow} \prod_{s(a)=v} \mathcal{F}^{+}(t(a)) \rightarrow 0
$$

is a split short exact sequence of right $R$-modules.

Definition 6.8. An object $M$ of a monoidal Grothendieck category $\mathcal{A}$ (see, for example, [15, pg.157] for the definition of a monoidal category) is said to be Gorenstein flat if there is an exact 
sequence

$$
\cdots \rightarrow F_{-3} \rightarrow F_{-2} \rightarrow F_{-1} \rightarrow F_{0} \rightarrow F_{1} \rightarrow F_{2} \rightarrow \cdots
$$

of flat objects such that $M=\operatorname{Ker}\left(F_{0} \rightarrow F_{1}\right)$ and such that the sequence is $E \otimes$-exact for every injective object $E$ (i.e. the functor $E \otimes-$ leaves the sequence exact).

Lemma 6.9. Let $Q$ be a left rooted quiver and $R$ be a Gorenstein ring. Then if $\mathcal{M}$ is a Gorenstein flat representation of $Q$, the representation $\mathcal{M}^{+}$of the quiver $Q^{o p}$ is Gorenstein injective.

Proof. Since $\mathcal{M}$ is Gorenstein flat, there exists an exact sequence

$$
\cdots \rightarrow \mathcal{F}_{-3} \rightarrow \mathcal{F}_{-2} \rightarrow \mathcal{F}_{-1} \rightarrow \mathcal{F}_{0} \rightarrow \mathcal{F}_{1} \rightarrow \mathcal{F}_{2} \rightarrow \cdots
$$

of flat representations with $\mathcal{M}=\operatorname{Ker}\left(\mathcal{F}_{0} \rightarrow \mathcal{F}_{1}\right)$ which remains exact when applying $\mathcal{E} \otimes-$, for all injective representation in $(Q, \operatorname{Mod}-R)$, but then by Corollary 6.7 we have the exact sequence

$$
\cdots \rightarrow \mathcal{F}_{2}^{+} \rightarrow \mathcal{F}_{1}^{+} \rightarrow \mathcal{F}_{0}^{+} \rightarrow \mathcal{F}_{-1}^{+} \rightarrow \mathcal{F}_{-2}^{+} \rightarrow \cdots
$$

of injective representations, such that $\mathcal{M}=\operatorname{Ker}\left(\mathcal{F}_{-1}^{+} \rightarrow \mathcal{F}_{-2}^{+}\right)$. We see that the last exact sequence is $\operatorname{Hom}(\mathcal{E},-)$ exact, for all injective representation $\mathcal{E}$. So let $\mathcal{E}$ be an injective representation. From (6.2) we have an exact

$$
\cdots \rightarrow \mathcal{E} \otimes \mathcal{F}_{-2} \rightarrow \mathcal{E} \otimes \mathcal{F}_{-1} \rightarrow \mathcal{E} \otimes \mathcal{F}_{0} \rightarrow \mathcal{E} \otimes \mathcal{F}_{1} \rightarrow \mathcal{E} \otimes \mathcal{F}_{2} \rightarrow \cdots
$$

so we have an exact sequence

$$
\cdots \rightarrow\left(\mathcal{E} \otimes \mathcal{F}_{2}\right)^{+} \rightarrow\left(\mathcal{E} \otimes \mathcal{F}_{1}\right)^{+} \rightarrow\left(\mathcal{E} \otimes \mathcal{F}_{0}\right)^{+} \rightarrow\left(\mathcal{E} \otimes \mathcal{F}_{-1}\right)^{+} \rightarrow\left(\mathcal{E} \otimes \mathcal{F}_{-2}\right)^{+} \rightarrow \cdots
$$

in $Q^{o p}$. But the canonical isomorphism

$$
\left(\mathcal{E} \otimes_{R Q} \mathcal{F}_{i}\right)^{+} \cong \operatorname{Hom}_{R Q}\left(\mathcal{E}, \mathcal{F}_{i}^{+}\right), i \in \mathbb{Z}
$$


still holds for rings without unit (as the case of $R Q$ whenever $Q$ has an infinite number of vertices) so we have the exact sequence

$$
\cdots \rightarrow \operatorname{Hom}\left(\mathcal{E}, \mathcal{F}_{2}^{+}\right) \rightarrow \operatorname{Hom}\left(\mathcal{E}, \mathcal{F}_{1}^{+}\right) \rightarrow \operatorname{Hom}\left(\mathcal{E}, \mathcal{F}_{0}^{+}\right) \rightarrow \operatorname{Hom}\left(\mathcal{E}, \mathcal{F}_{-1}^{+}\right) \rightarrow \operatorname{Hom}\left(\mathcal{E}, \mathcal{F}_{-2}^{+}\right) \rightarrow \cdots
$$

Lemma 6.10. Let $Q$ be a forest whose connected components are barren trees and ${ }_{R} R$ be noetherian. Then a representation $\mathcal{E}$ of $Q$ is injective if and only if $\mathcal{E}^{+}$is flat (over $Q^{o p}$ )

Proof. We will use the characterization of flat representations given in [9, Theorem 3.7] and Corollary 5.5. Notice that, for all $v \in V, \mathcal{E}(v)$ is an injective $R$-module if and only if $\mathcal{E}^{+}(v)$ is a flat $R$-module (see for example [8, Corollary 3.2.17]) and furthermore the sequence

$$
0 \rightarrow \mathcal{K}(v) \rightarrow \mathcal{E}(v) \rightarrow \prod_{s(a)=v} \mathcal{E}(t(a)) \rightarrow 0
$$

is exact, with $\mathcal{K}(v)$ injective if and only if

$$
0 \rightarrow \oplus_{t(a)=v} \mathcal{E}^{+}(s(a)) \rightarrow \mathcal{E}^{+}(v) \rightarrow \mathcal{K}^{+}(v) \rightarrow 0
$$

is an exact sequence with $\mathcal{K}^{+}(v)$ flat (so hence pure exact sequence) for all $v \in V$. Notice that, since the connected components of $Q$ are barren trees, then, for every vertex $v \in V$ the set $\{a \in \Gamma: s(a)=v\}$ is finite.

Proposition 6.11. Let $Q$ be a forest whose connected components are barren trees and let $R$ be Gorenstein. A representation $\mathcal{M}$ of the quiver $Q$ is Gorenstein flat if and only if for each vertex $v, \mathcal{M}(v)$ is a Gorenstein flat $R$-module and the sequence

$$
0 \rightarrow \oplus_{t(a)=v} \mathcal{M}(s(a)) \stackrel{f_{v}}{\longrightarrow} \mathcal{M}(v) \rightarrow \mathcal{C}(v) \rightarrow 0
$$


is exact and $\mathcal{C}(v)$ is Gorenstein flat (where $f_{v}: \oplus_{t(a)=v} \mathcal{M}(s(a)) \rightarrow \mathcal{M}(v)$ is a morphism induced by $\mathcal{M}(s(a)) \rightarrow \mathcal{M}(v))$.

Proof. Necessity. By Lemma 6.9, $\mathcal{M}^{+}$is Gorenstein injective, so by Corollary $6.3 \mathcal{M}^{+}(v)$ is a Gorenstein injective $R$-module for all $v \in V$, but then $\mathcal{M}(v)$ is a Gorenstein flat $R$-module. Furthermore since

$$
0 \rightarrow \mathcal{C}^{+}(v) \rightarrow \mathcal{M}^{+}(v) \rightarrow \prod_{s(a)=v} \mathcal{M}^{+}(t(a)) \rightarrow 0
$$

is exact with $\mathcal{C}^{+}(v)$ Gorenstein injective, then

$$
0 \rightarrow \oplus_{t(a)=v} \mathcal{M}(s(a)) \rightarrow \mathcal{M}(v) \rightarrow \mathcal{C}(v) \rightarrow 0
$$

is exact with $\mathcal{C}(v)$ Gorenstein flat, for every $v \in V$.

Sufficiency. Since $R$ is Gorenstein the hypothesis is equivalent to saying that $\mathcal{M}^{+}(v)$ is Gorenstein injective and that

$$
0 \rightarrow \mathcal{C}^{+}(v) \rightarrow \mathcal{M}^{+}(v) \rightarrow \prod_{s(a)=v} \mathcal{M}^{+}(t(a)) \rightarrow 0
$$

is exact with $\mathcal{C}^{+}(v)$ Gorenstein injective. But, by Corollary 6.3, this implies that $\mathcal{M}^{+}$is a Gorenstein injective representation. So there exists an exact sequence

$$
\mathcal{E}_{n} \rightarrow \mathcal{E}_{n-1} \rightarrow \cdots \mathcal{E}_{0} \rightarrow \mathcal{M}^{+} \rightarrow 0
$$

with $\mathcal{E}_{i}$ injective representations for all $i$ such that $1 \leq i \leq n$. Now, by Lemma 6.10,

$$
0 \rightarrow \mathcal{M}^{++} \rightarrow \mathcal{E}_{0}^{+} \rightarrow \cdots \rightarrow \mathcal{E}_{n-1}^{+} \rightarrow \mathcal{E}_{n}^{+}
$$

is an exact sequence of flat representations. $\operatorname{So} \operatorname{Tor}^{i}\left(\mathcal{L}, M^{++}\right)=0$, for all $i \geq 1$ and for all representation $\mathcal{L}$ such that $\operatorname{inj}_{\operatorname{dim}} \mathcal{L}<\infty$. But now there exists a pure exact sequence

$$
0 \rightarrow \mathcal{M} \rightarrow \mathcal{M}^{++} \rightarrow \mathcal{M}^{++} / \mathcal{M} \rightarrow 0
$$


of representations, so $\operatorname{Tor}^{1}\left(\mathcal{L}, \mathcal{M}^{++} / \mathcal{M}\right)=0$ for all such $\mathcal{L}$. But then $\operatorname{Tor}^{i}\left(\mathcal{L}, \mathcal{M}^{++} / \mathcal{M}\right)=0$, for every $i \geq 1$. So then $\operatorname{Tor}^{i}(\mathcal{L}, \mathcal{M})=0$ for each $i \geq 1$ and for all representations $\mathcal{L}$ such that $\operatorname{inj}_{\operatorname{dim}_{Q}} \mathcal{L}<\infty$. But this means that if we take a complex

$$
\cdots \rightarrow \mathcal{F}_{-2} \rightarrow \mathcal{F}_{-1} \rightarrow \mathcal{F}_{0} \rightarrow \mathcal{F}_{1} \rightarrow \mathcal{F}_{2} \rightarrow \cdots
$$

such that $\mathcal{M}=\operatorname{Ker}\left(\mathcal{F}_{0} \rightarrow \mathcal{F}_{1}\right)$ with $\mathcal{F}_{i}$ a flat representation for all $i \in \mathbb{Z}$, then this will be $\mathcal{E} \otimes-$

exact for all injective representation $\mathcal{E}$. Furthermore, by [5, Theorem 3.7], $\operatorname{inj}_{\operatorname{dim}} R Q R Q<\infty$ so then the complex will be exact.

\section{ACKNOWLEDGEMENTS}

The authors wish to thank the referee for many helpful comments and suggestions.

\section{REFERENCES}

[1] I. Assem, D. Simson and A. Skowronski. Elements of the representation theory of associative algebras. Vol. 1. Techniques of representation theory. London Mathematical Society Student Texts, 65. Cambridge University Press, Cambridge, 2006.

[2] E. Enochs and S. Estrada. Projective representations of quivers. Comm. Algebra. 33(2005), 3467-3478.

[3] E. Enochs and S. Estrada Relative homological algebra in the category of quasi-coherent sheaves. Adv. in Math. 194 (2005), 284-295.

[4] E. Enochs, S. Estrada and J.R. García Rozas. Gorenstein Categories. Tate Cohomology on Projective Schemes. To appear in Math. Nachr.

[5] E. Enochs, S. Estrada, J.R. García Rozas and A. Iacob. Gorenstein representations of quivers. To appear in Arch. Math. (Basel).

[6] E. Enochs, J.R. García Rozas, L. Oyonarte and S. Park. Noetherian Quivers. Quaest. Math. 25(4) (2002), 531-538.

[7] E. Enochs and I. Herzog. A homotopy of quiver morphisms with applications to representations. Canad. J. Math. 51(2) (1999), 294-308.

[8] E. Enochs and O. Jenda. Relative homological algebra. De Gruyter Expositions in Mathematics 30, de Gruyter, Berlin, 2000.

[9] E. Enochs, L. Oyonarte and B. Torrecillas. Flat covers and flat representations of quivers. Comm. Algebra. 32(4)(2004), 1319-1338.

[10] S. Estrada. Monomial algebras over infinite quivers. Applications to $N$-complexes of modules. To appear in Comm. Algebra.

[11] P. Gabriel. Unzerlegbare Darstellungen I. Manuscripta Math. 6 (1972), 71-103.

[12] M. Hovey. Model Categories. AMS, Mathematical Surveys and Monographs, 63, 1999.

[13] Y. Iwanaga. On rings with finite self-injective dimension. Comm. Algebra. 7(4) (1979), 393-414.

[14] L. Le Bruyn and C. Procesi. Semisimple representations of quivers. Trans. Amer. Math. Soc. 317(2) (1990), 585-598.

[15] S. Mac Lane. Categories for the working mathematician., 2nd. ed., Graduate Texts in Mathematics 5, Springer-Verlag, New York, 1998.

[16] B. Stenström. Rings of quotients. Springer-Verlag, 1975. 
Department of Mathematics, University of Kentucky, Lexington, Kentucky 40506-0027, U.S.A.

E-mail address: enochs@ms.uky.edu

Departamento de Matemática Aplicada, Universidad de Murcia,Campus del Espinardo, Espinardo (Murcia) 30100, Spain

E-mail address: sestrada@um.es

Departamento de Álgebra y A. Matemático, Universidad de Almería, Almería 04071, Spain

E-mail address: jrgrozas@ual.es 